\title{
Analytical modeling of sub-surface porous reservoir compaction
}

\author{
Tom A. Jelmert ${ }^{1} \cdot$ Tommy Toverud $^{1}$ \\ Received: 20 December 2017 / Accepted: 10 May 2018 / Published online: 11 June 2018 \\ (c) The Author(s) 2018
}

\begin{abstract}
Fluid extraction has led to compaction, land subsidence, flooding and even earthquakes many places around the world. Although rare, the environmental costs could be overwhelming. Once compaction has been identified, possible future consequences should be investigated. If deemed adverse, preventive actions should be started as soon as possible to mitigate future damage. The purpose of this study is to enable simultaneous prediction of possible changes in thickness and permeability by a simplified analytical model. These changes may occur simultaneously or separately. Although best, studies by numerical simulation are time consuming and expensive. A fluid extraction period may also be necessary to match the model to observed behavior. A pressure transient test, on the other hand, may be conducted once a formation has been penetrated. Due to simplicity and ease of application, we believe our methodology will be useful, at least as screening tool. Our model, which is a generalization of a classical one, Raghavan et al. (SPEJ 253:267-386, 1972) and Pedrosa (1986), has been extended to account for boundary dominated flow and for the effect of wellbore storage and skin. During the last decades, many studies have expanded and improved the Pedrosa theory. We rediscover the Pedrosa (1986) equations, but with a composite (sum) modulus replacing the permeability modulus and an additional modulus to thickness in the storability coefficient. Our model will simplify to the Pedrosa model and many others by simple changes in the input data. The traditional well test model, without stress-sensitivity, is included as limiting behavior. Most commercial well test simulators already include the majority of coding to take advantage of the proposed technique. We find that the value of the sum modulus may either enhance or mask the existence of stress-sensitivity. The latter may be an important problem in case of a negative permeability modulus. This could reduce the value of the sum modulus due to appearance of additional fractures. Then, results obtained with the present model are likely to be misleading. The present methodology should be used with caution under this condition. Important in situ fracture generation may be detected by strong micro seismic activity. Core analysis may also give a forewarning of brittle formations. The use of the proposed well test model leads to many different possible interpretations (non-uniqueness). Hence, selecting a plausible reservoir model depends on the existence of information that is independent of well testing. The proposed model may be of interest in ground water hydrology, for wastewater disposal, geothermal- and petroleum reservoirs. We derive equations for the dynamic behavior of the thickness and permeability.
\end{abstract}

Keywords Compaction - Analytical solutions $\cdot$ Compaction ramifications $\cdot$ Type curves

\section{Introduction}

Fluid extraction has led to compaction, land subsidence, flooding and even earthquakes many places around the world. Decreasing thickness and altered permeability may

Tom A. Jelmert

tom.aage.jelmert@ntnu.no

Tommy Toverud

ttoveru@hotmail.com

1 Norwegian University of Science and Technology, NTNU, 7094 Trondhem, Norway compromise well integrity and have serious environmental ramifications. On the positive side, compaction may also be an important production mechanism. Examples of subsidence are Venice, Groningen and Ekofisk, Doornhof et al. (2006). Once compaction has been identified, possible future consequences should be estimated. If deemed adverse, preventive actions should be taken as soon as possible to mitigate future damage. Subsidence due to fluid withdrawal "has been measured to about $10 \mathrm{~m}$ in Mexico, Japan and the United States for example", Johnson (1991).

Manmade subsidence has been with us for more than a century, Prat and Johnson (1926). The problem has not 
yet been satisfactorily solved. It still reoccurs many places around the world.

Subsidence is often reported in the regular press. For example, Guardian reported subsidence in Groningen, (10th October, 2015) and New York Times about Ekofisk, (August 19, 1987), both in Europe.

The focus of this work is on simultaneous changes in reservoir thickness and permeability.

Doornnhof et al. (2006) point out that a variety of differently classified reservoirs may be prone to compaction and subsidence. Examples are; high and low permeability reservoirs, geo-pressured reservoirs, etc.

Stress-sensitive reservoirs may also be classified as non-recoverable and/or recoverable. A recoverable (elastic) reservoir will rebound if refilled. Whatever classification, we assume that the pressure behavior may be described by exponential functions.

The cost effective way to achieve dynamic reservoir data is pressure transient analysis (PTA). A test may be conducted once a new well has penetrated a formation. In addition, pressure transients move quickly compared to fluid movements. Hence, a well test may give an early warning of incipient problems.

Exploration wells are typically vertically drilled to delineate the reservoir thickness, extent and fluid content. Knowledge of the initial thickness is crucial to quantify deformation, reservoir volume etc.

A host of studies on the effect of stress-sensitivity has been published. Research on the combined effect of changes in permeability and thickness has not received much attention. In many cases, there is a contrast in adjustment time. The effect of permeability changes (reduced inflow performance) can show up quickly, while compaction requires more time. The rate of withdrawal, which is crucial for the well economy, is a strong function of the current value of the permeability. Hence, the variation of permeability is more likely to receive attention.

We invoke the traditional assumptions: The volume of grains remain constant, all pressure-dependent variables may be described by exponential functions of pressure and, in case of build-up, negligible hysteresis. In addition, the assumption of an instantaneous response is implied. Next, we assume that the pressure dependency of the thickness also follows an exponential pressure function. The latter assumption leads to an additional "compressibility" in the diffusivity equation.

An inherent problem of traditional well test interpretation is non-unique responses and more variables than equations. Hence, the analyst has to rely on data from other sources. Core analysis, well logging, PVT analysis etc. may help estimate the initial rock properties: permeability, porosity and thickness. In addition, fluid and matrix compressibility may be obtained. These techniques are useful, also for stress-sensitive reservoirs.

Pressure transient analysis depends on matching predictions from a plausible mathematical model to observed data. The model is a diffusivity equation with appropriate boundary conditions. In case of stress-sensitive reservoirs, the diffusivity equation is non-linear. This is because coefficients to the gradient terms depends on pressure changes, Eq. (1). The non-linear diffusivity equation has no known exact solution.

Approximate solutions, however, may be available, AlHussainy et al. (1966). They proposed to transform the non-linear diffusivity equation into a simpler form by the Kirchhoff integral. The result was a diffusivity equation of linear appearance. The simplified equation, however, was still non-linear. This problem was avoided (neglected) by evaluating all pressure-dependent variables at the initial condition. Then, they obtained a linear equation in terms of transformed variable. The important consequence is that many known solutions for slightly compressible fluids are also available for gas wells. They coined the term "real gas pseudo potential" to describe their transformed variable. Then, the pdfunction for a traditional reservoir is also a solution to the pseudo potential function. Since then, the pseudo potential technique has been applied to PTA for many important problems, including stress-sensitive reservoirs.

Raghavan et al. (1972) proposed a pioneering well test model for pressure-dependent rock and fluid properties. They used the pseudo pressure approach. Their pseudo potential function included the effect of density, permeability and porosity changes.

Literature shows that most analytical studies rely on either the pseudo pressure approach or a logarithmic substitution (Cole-Hopf transformation). Jelmert and Selseng (1997) showed that these are equivalent for exponential pressure behavior. The authors pointed out that the former is more flexible with respect to the choice of parametric equations. Use of the former technique has been discussed in many studies, for example, Friedel and Voigt (2009) and Samaniego and Cinco-Ley (1989).

Pedrosa (1986) used a similar approach as Raghavan et al. (1972), but he assumed an exponential relationship between permeability and pressure. In addition, he invoked the slightly compressible fluid assumption. With known initial value, the permeability-function depends the modulus only. A logarithmic substitution, Eq. (7), transforms the non-linear governing equation into a diffusivity equation of simpler appearance, Eq. (8). The non-linear diffusivity coefficient, however, makes analytical solutions intractable. Again, this problem may be circumvented by evaluation of all pressure-dependent variables at the initial condition. Then, the coefficient to the temporal derivative assumes unit value and Eq. (8) simplifies to the traditional diffusivity equation without stress-sensitivity, Eq. (47). To improve 
an obviously simplistic solution, he proposed to enhance the accuracy by regular perturbations. He published two solutions. First, the zero order solution, which is the solution to the linear problem, the traditional line source solution, Eq. (48). Then, an improved solution, the first order perturbation, Eq. (49). Once an approximate solution has been obtained, it can easily be converted back to pressure by Eq. (42). This is advantageous since pressure, not pseudo pressure, is measured.

Kikani and Pedrosa (1991) matched the Pedrosa (1986) model to real data. They argued that the zero order solution is of sufficient accuracy for many engineering applications. This is because the first order solution is multiplied by the dimensionless permeability modulus squared, which usually assumes small values. In addition, they showed that the permeability modulus could be quantified by type-curve analysis, provided the initial permeability may be obtained. They also obtained a second order perturbation solution. According to them, the latter did not make much difference.

During the last decades, many studies have expanded and improved the Pedrosa theory (1986). Our model will simplify to the Pedrosa (1986) model and many others by simple changes in the input data.

\section{Theory}

The basic theory of this study has been explained previously, Jelmert and Toverud (2016). They found that the pressure equations for a compacting reservoir remain unchanged when compared against those obtained in Kikani and Pedrosa (1991), but with dimensionless time differently defined. In addition, the composite (sum) modulus replaces the permeability modulus. This study expands the scope of their methodology.

The diffusivity equation, Eq. (1), may be thought of as consisting of two parts, the transport term on the left hand side (LHS) and the storage term on the right hand side (RHS). The mass flow rate shows up inside the parenthesis on LHS. The outer space derivative implies change of mass flow rate. The storability is the coefficient to the time derivative on the RHS. The terms inside the parenthesis is the mass currently in place. The derivative with respect to pressure implies rate of change. Hence, the rate of change in mass flow rate is balanced by the rate of change of mass stored.

The generalized diffusivity equation for compacting media is, Raghavan et al. (1972):

$$
\begin{gathered}
\frac{1}{r} \frac{\partial}{\partial r}\left(\frac{2 \pi \rho_{i} k_{i} h_{i}}{\mu_{i}} \frac{\rho_{n}(\Delta p) k_{n}(\Delta p) h_{n}(\Delta p)}{\mu_{n}(\Delta p)} r \frac{\partial \Delta p}{\partial r}\right) \\
\quad=\frac{\partial}{\partial \Delta p}(h(\Delta p) \rho(\Delta p) \varphi(\Delta p)) \frac{\partial \Delta p}{\partial t},
\end{gathered}
$$

On LHS, the permeability-thickness product accounts for altered flow capacity. On RHS, the inclusion of thickness accounts for the shrinking reservoir volume due to compaction.

Index $n$ denotes normalized to the initial condition, while index $i$ denotes at the initial condition. The pressure-dependent variables are; density, permeability, thickness and viscosity on LHS. On RHS: thickness, density and porosity. The new element, compared to previous studies, is the simultaneous appearance of permeability and thickness as state variables.

After some manipulations of Eq. (1), we find:

$\frac{1}{r} \frac{\partial}{\partial r}\left(e^{-\tau \Delta p} r \frac{\partial \Delta p}{\partial r}\right)=\frac{\varphi_{i} \mu_{i}}{k_{i}} e^{-\left(\xi+c_{\ell}+c_{\mathrm{ma}}\right) \Delta p}\left\{\xi+c_{\ell}+c_{\mathrm{ma}}\right\} \frac{\partial \Delta p}{\partial t}$,

where

$\tau=\gamma+\xi-v+c_{\ell}$.

The addends of the composite modulus are modulus to permeability, thickness, viscosity, and the compressibility of the fluid. The addition property depends on the assumption of exponential pressure functions.

Pedrosa (1986) found that the perturbation procedure would be simplified whenever Eq. (4) is a valid approximation to Eq. (2). This criterion is satisfied if $\gamma>>c_{\ell}+c_{\mathrm{ma}}$. They considered flow of slightly compressible fluids.

$\frac{1}{r}\left\{\frac{\partial}{\partial r}\left(r \frac{\partial \Delta p}{\partial r}\right)-\tau r\left(\frac{\partial \Delta p}{\partial r}\right)^{2}\right\}=\frac{\varphi_{i} \mu_{i}}{k_{i}} e^{\tau \Delta p}\left\{\xi+c_{\ell}+c_{m a}\right\} \frac{\partial \Delta p}{\partial t}$.

The dimensionless equivalent of Eq. (4) becomes:

$\frac{1}{r_{\mathrm{D}}}\left\{\frac{\partial}{\partial r_{\mathrm{D}}}\left(r_{\mathrm{D}} \frac{\partial p_{\mathrm{D}}}{\partial r_{\mathrm{D}}}\right)-\tau_{\mathrm{D}} r_{\mathrm{D}}\left(\frac{\partial p_{\mathrm{D}}}{\partial r_{\mathrm{D}}}\right)^{2}\right\}=e^{\tau_{\mathrm{D}} p_{\mathrm{D}}} \frac{\partial p_{\mathrm{D}}}{\partial t_{\mathrm{D}}}$.

We invoke the same assumption \{Eq. (2) may be approximated by Eq. (4)\}, but the simplification is different in detail. For a positive permeability modulus, the criterion of validity becomes:

$\gamma_{\mathrm{D}}>>c_{\mathrm{Dma}}+v_{\mathrm{D}}$.

Again, the condition of a slightly compressible fluid is implied. As pointed out in Appendix B, the dimensionless moduli are proportional to the real ones.

Pedrosa (1986) proposed a logarithmic transformation.

$p_{\mathrm{D}}\left(r_{\mathrm{D}}, t_{\mathrm{D}}\right)=-\frac{1}{\tau_{\mathrm{D}}} \ln \left(1-\tau_{\mathrm{D}} \eta\left(r_{\mathrm{D}}, t_{\mathrm{D}}\right)\right)$.

Substitution of Eq. (7) into Eq. (5) yields:

$\frac{\partial^{2} \eta}{\partial r_{\mathrm{D}}^{2}}+\frac{1}{r_{\mathrm{D}}} \frac{\partial \eta}{\partial r_{\mathrm{D}}}=\frac{1}{1-\tau_{\mathrm{D}} \eta} \frac{\partial \eta}{\partial t_{\mathrm{D}}}$ 
The transformed variable (pseudo pressure) is denoted by $\eta$.

We obtain the same equations as Kikani and Pedrosa (1991), i.e., Equation (8). Hence, the same perturbation technique and solutions are available for compacting reservoirs. The perturbation technique is well known and will not be repeated here. Suffice to say that the perturbation schemes starts out with the linearized (zero order perturbation) version of Eq. (8), i.e., the coefficient to the temporal derivative is unity.

In line with the majority of PTA studies, dimensionless time is defined to include the total compressibility as a factor in the denominator. Hence, the generalized dimensionless time includes the modulus to thickness, $\xi$. This is because shrinking reservoir volume, due to compaction, leads to decreased storability.

$t_{\mathrm{D}}=\frac{k_{i} t}{\varphi_{i} \mu_{i}\left(\xi+c_{\ell}+c_{\mathrm{ma}}\right) r_{w}^{2}}$.

Suppose all other variables are known from other sources, then the modulus to thickness may be obtained from the "time match", Jelmert and Toverud (2016).

In appendix A we show that the exponential term, LHS of Eq. (2), is akin to normalized transmissibility, $T_{n}(\Delta p)=e^{-\tau \Delta p}$, where the pressure change is $\Delta p=p_{i}-p$. Then, the dimensionless model (boundary conditions and differential equation) will reduce to Eq. (46) and Eq. (47). A reduced value of $\tau$ leads to decreased apparent stress- sensitivity (decreased transmissibility). For an increase, it is the other way around. For example, the permeability modulus may assume a negative value since new fractures and fissures may be created. Then, there is a chance that the permeability- and the viscosity modulus in Eq. (3) lead to a small sum, which in turn could mask real stress-sensitivity.

In many cases, the zero order solution is of sufficient accuracy. Then, the best way to proceed is to evaluate the pressure-dependent coefficient to the temporal derivative of Eq. (2) at the initial condition. Under this assumption, a large but constant fluid compressibility is properly accounted for.

The set of assumptions include instantaneous responses, which may not be realistic for all reservoirs. If the response time is long compared to the duration of the test, then possible stress-sensitivity will be impossible to detect. This does not mean that the proposed method is useless. The moduli may be estimated from core analysis or experience from other wells in this or similar formations. Then, the model can be used as a tool to obtain some information about the expected future behavior.

The Laplace space solutions technique has proved to be a valuable tool in PTA, van Everdingen and Hurst (1949). Many governing equations and boundary conditions remain unchanged during the pseudo pressure transformation. Hence, these solutions are also available for pressure sensitive reservoirs, but in terms of the pseudo pressure.

Due to linearity, Eqs. (46) and (47), the effect of wellbore storage may be computed convolution:

$\bar{\eta}_{w v 0}(s)=-\frac{s \bar{\eta}_{c 0}(s)+S}{s\left(1+C_{\mathrm{D}} s^{2} \bar{\eta}_{c 0}+s C_{\mathrm{D}} S\right)}, \quad r_{\mathrm{D}}=1$.

Indices $w, v, c$ and 0 denotes well, variable rate, constant rate and zero order perturbation, respectively. Variables $C_{D}, S$ and $s$ denotes wellbore storage-, skin factor and the Laplace space variable respectively. A Laplace transformed variable is indicated by a bar on top. Equation (10) may be inverted numerically.

$p_{\mathrm{D}}\left(r_{D w}, t_{\mathrm{D}}\right)=-\frac{1}{\tau_{\mathrm{D}}}\left(1-\tau_{\mathrm{D}} L^{-1}\left\{\bar{\eta}_{w 0}\right\}\right)$.

The bounded reservoir responses, with a no-flow boundary, may be calculated by the traditional equation.

$\bar{\eta}_{0}\left(r_{\mathrm{D}}, s\right)=\frac{K_{1}\left(r_{\mathrm{eD}} \sqrt{s}\right) I_{0}\left(r_{\mathrm{D}} \sqrt{s}\right)+I_{1}\left(r_{\mathrm{eD}} \sqrt{s}\right) K_{0}\left(r_{\mathrm{D}} \sqrt{s}\right)}{s^{3 / 2}\left(I_{1}\left(r_{\mathrm{eD}} \sqrt{s}\right) K_{1}(\sqrt{s})-K_{1}\left(r_{\mathrm{eD}} \sqrt{s}\right) I_{1}(\sqrt{s})\right)}$,

$K, I$ are modified Bessel functions. Indices 1 and 2 denote first and second kind, respectively. $r_{e}$ is the distance to the external boundary. The effect of skin and wellbore storage has been neglected, in Eq. (12).

The thickness and permeability depend on the fluid pressure. Under the assumption of exponential pressure behavior, Jelmert and Toverud (2016) found.

$h_{n 0 s f}\left(t_{\mathrm{D}}\right)=\left\{1-\tau_{\mathrm{D}} \eta_{0 s f}\right\}^{\frac{\xi_{\mathrm{D}}}{\tau_{\mathrm{D}}}}$,

$k_{n 0 s f}\left(t_{\mathrm{D}}\right)=\left\{1-\tau_{D} \eta_{0 s f}\right\}^{\frac{\gamma_{\mathrm{D}}}{\tau_{\mathrm{D}}}}$

Indices, $n, 0$ and $s f$ denote normalized with respect to the initial condition, 0 -order perturbation and sand face, respectively. The sand face is located at the wellbore, but on the reservoir side of the skin.

As pointed out previously, $\tau_{D}$ may be obtained as a parameter on the type curve and $\xi_{\mathrm{D}}$ from the "time Match". The dimensional equivalents are readily available.

$h_{0 s f}\left(t_{\mathrm{D}}\right)=h_{i}\left\{1-\tau_{\mathrm{D}} \eta_{0 s f}\right\}^{\frac{\xi}{\tau}}$,

$k_{n 0 s f}\left(t_{\mathrm{D}}\right)=k_{i}\left\{1-\tau_{\mathrm{D}} \eta_{0 s f}\right\}^{\frac{\gamma}{\tau}}$. 


\section{Results}

Many interpretation techniques depend on the assumption of a constant flowrate. This condition may be difficult to achieve for a sufficient length of time. A pressure buildup (PBU) test mitigates this problem in a simple way by closing the well. Traditionally, the PBU has been analyzed contingent to the assumption of two concurrent radial flow periods in the superposition function. The flowrate depends on the permeability thickness product. The flow capacity is best estimated by analysis of the infinite-acting radial flow period. We consider infinite-acting radial flow, without wellbore storage and skin. The traditional Horner equation is included in the generalized solution as limiting behavior, Jelmert and Toverud (2016), see Eq. (55). The Horner equation shows up as the semi-log straight line (in red) on the dimensionless semi-log plot in Fig. 1. Figure 1 is the same as Fig. 5 in Kikani and Pedrosa (1991). In Figs. 1 and 2 we invoke Eq. (4) to facilitate perturbations. Both the Kikani and Pedrosa (1991) and Jelmert and Toverud (2016) models may be matched to the same well test data. The existence of multiple interpretations (non-uniqueness) is problematic. This topic will be discussed later.

The non-linear correction shows up as the vertical displacement between the non-linear (broken) and linear (solid) curve. The divergence increases with increasing values of the sum modulus and with time. Failure to recognize stresssensitivity may lead to erroneous well test interpretation.

Figure 1 shows that, in this case, the effect of the first order perturbation is negligible. Due to the Horner ratio on the horizontal axis, the effect of a possible change in thickness is lost. A better approach is to plot pressure against the shut-in time. This technique has been called the MDH method, Matthews, Brons and Hazebrook (1954). Then, it is possible to compute the dimensionless modulus to thickness from the "time match", Jelmert and Toverud (2016).

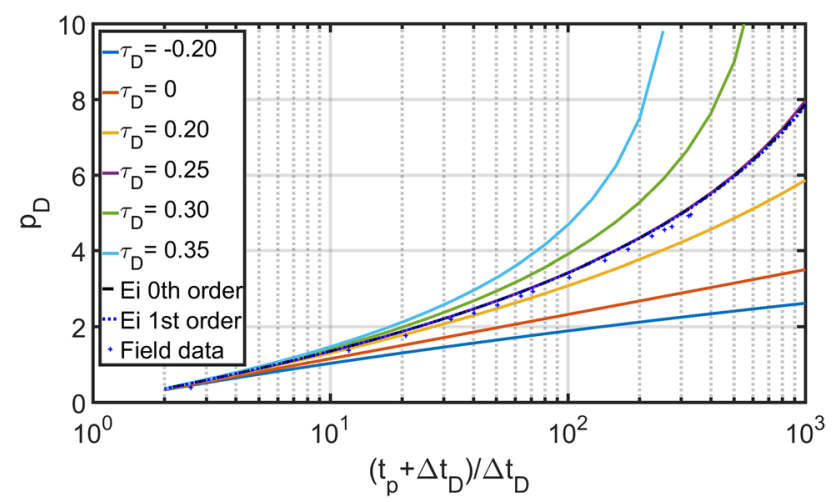

Fig. 1 Comparison of zero and first order perturbation, Horner ratio on the horizontal axis

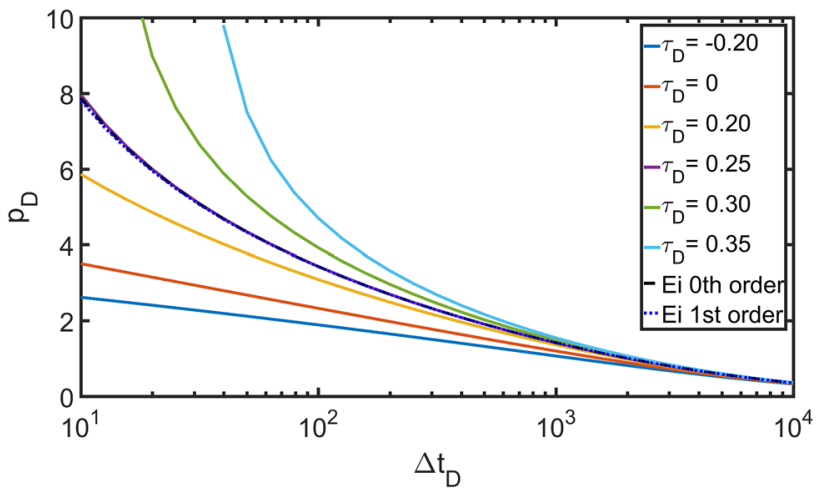

Fig. 2 MDH plot, Infinite-acting radial flow

The semi-log MDH type curves are shown in Fig. 2 . Both Figs. 1 and 2 show that the case with negative sum modulus falls below the zero value response. The responses, $\left(\tau_{\mathrm{D}}=0.2\right.$ and $\left.\tau_{\mathrm{D}}=-0.2\right)$ are not symmetrical about the zero value (red $\tau_{\mathrm{D}}=0$ ) straight line. This is a consequence of the non-linearity.

Suppose the numerical value of the viscosity modulus, $v$, is close to the total fluid compressibility, then Eq. (3) will reduce to:

$\tau \approx \gamma+\xi$.

If the effect of the modulus to thickness is neglected, either by error or on purpose, then the generalized model will simplify to the Pedrosa (1986) model.

Extension of the generalized theory to the flow of a real gas is straight forward as shown in Pedrosa (1986). This is because the generalized model depends on the same governing equations.

Next, we include the effect of wellbore storage and skin. This effect has been discussed in Kikani and Pedrosa (1991), Jelmert and Toverud (2017a) and Cao et al. (2004).

Figure 3 shows the transition from wellbore storage into radial flow for $\tau_{D}=0.05$. The non-linear responses are indicated by broken curves. A conspicuous feature is that the logarithmic derivatives (lower curves) do not show up as a single horizontal line during radial flow. Rather than that, we see a family of upward bending curves. The linear case (solid curves) corresponds to the dimensionless pressure without stress-sensitivity or the zero order solution of the transformed variable.

Figure 4 shows the time response of the thickness normalized to its initial value. Index sf denotes sand face (at the wellbore but at the reservoir side of the skin) and index zero denotes a zero order perturbation.

The behavior can be explained as follows: Initially, there is negligible compaction since the reservoir flow rate is insignificant. The majority of the produced derives from the wellbore (unloading). Next comes a transition 


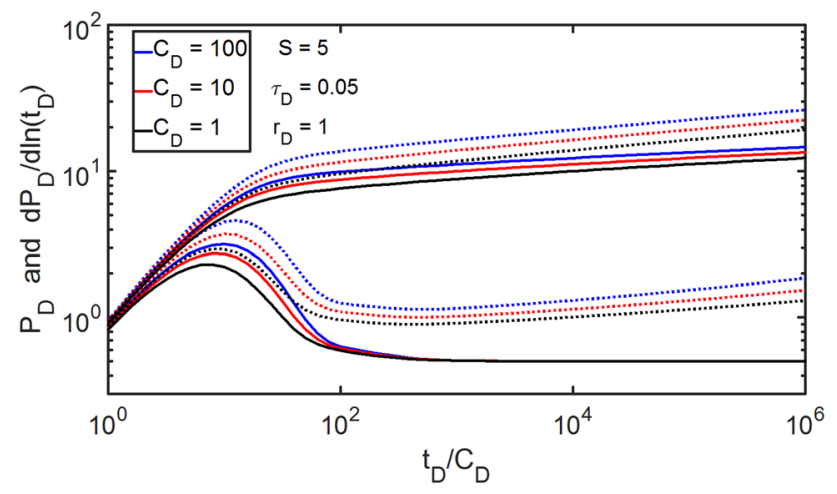

Fig. 3 Pressure and pressure derivative, non-linear (broken curves) and linear (solid curves) responses. From Jelmert and Toverud (2017a)

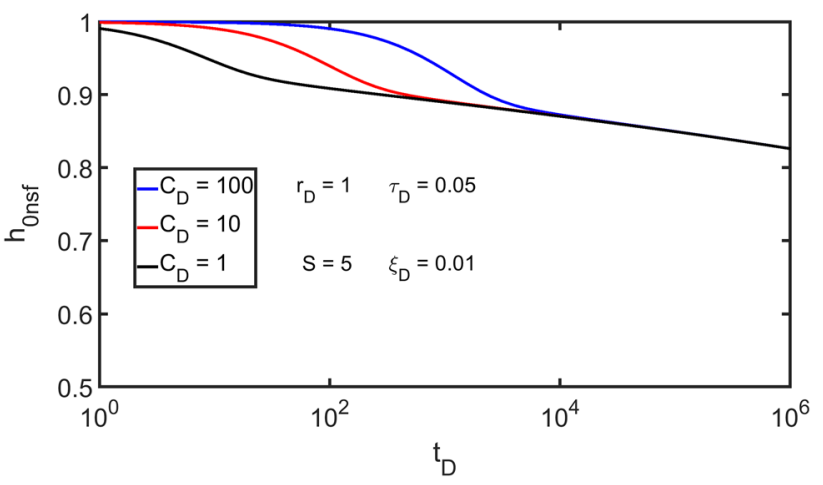

Fig. 4 Semi-log plot of normalized thickness as a function of dimensionless time. From Jelmert and Toverud (2017a)

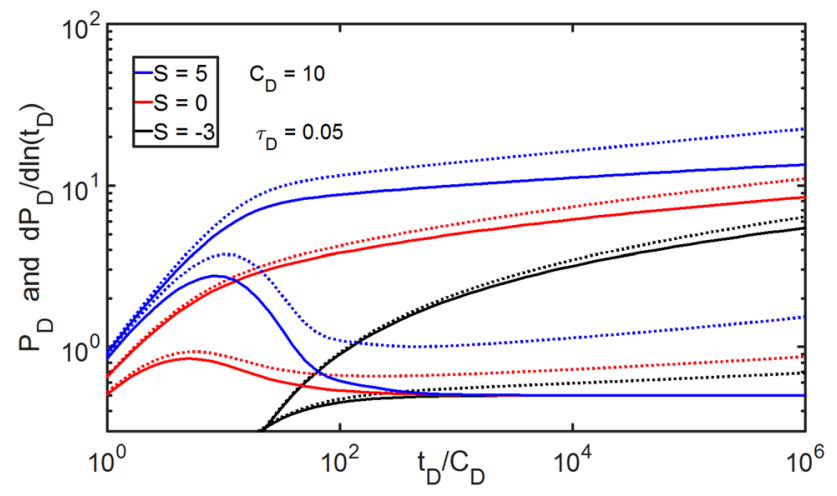

Fig. 5 Pressure and pressure derivative, non-linear (unbroken curves) and linear (solid) responses

period. The "almost" straight line corresponds to established reservoir flow.

For a large thickness, even a small decline in normalized thickness could lead to significant compaction. This

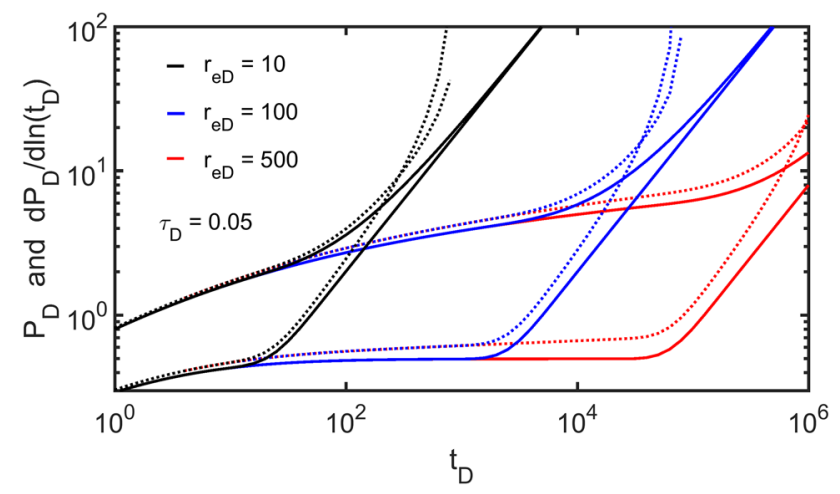

Fig. 6 Pressure and pressure derivative, non-linear (broken curves) and linear (solid curve) responses

is because the actual compaction depends on the initial thickness as a multiplication factor,

$h(\Delta p)=h_{i} h_{n}(\Delta p)$.

Figure 5 shows the effect of the skin factor. The three cases corresponds to damage (positive $S$ ), no damage $(S=0)$ and a stimulation (negative $S$ ). To generate the latter case, we invoked the concept of apparent wellbore radius (denoted by index wa), that is the pressure response is evaluated at an increased wellbore radius, $r_{\mathrm{wa}}=r_{\mathrm{w}} e^{-S}$.

Use of the equivalent radius is possible since a logarithmic function is a monotonically increasing function for arguments larger than zero. This property precludes multiple values of the equivalent radius.

Figure 6 shows the effect of a radial no-flow external boundary. Again, we observe a clear difference between the linear and non-linear cases. In the same way as a single horizontal line in the pressure derivative is a characteristic of radial flow, the unit slope lines in the linear responses indicate pseudo-steady state flow. None of the non-linear responses (broken curves) is of the pseudo-steady state type.

Boundary dominated flow starts with deviation of the responses from the almost semi-log straight line in Fig. 7. As expected, the infinite-acting period will last longer for a large external radius (Figs. 6, 7).

\section{Discussion}

An altered permeability could be caused by reduced pore radii, collapsing fractures or induction of new ones. The latter phenomenon may lead to a negative permeability modulus. Then, use of the present model may be misleading. Possible cracking of the formation may be detected by micro seismic activity. Hence, the proposed method should be used with caution under this condition. A reservoir with 


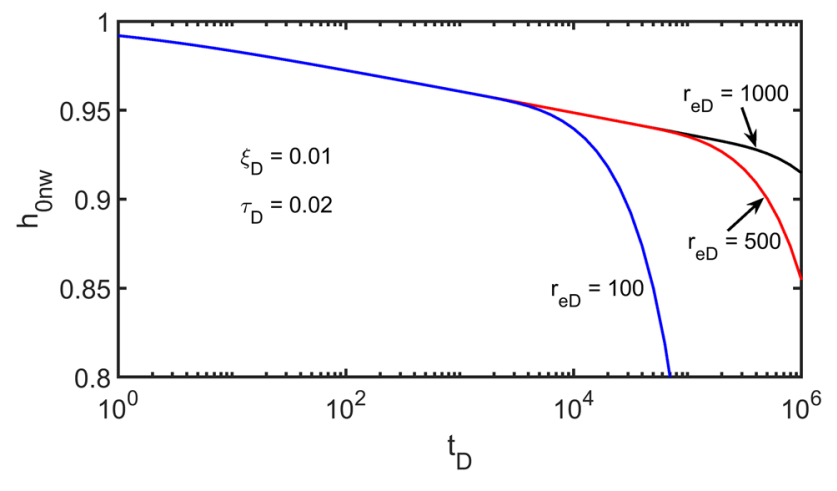

Fig. 7 Effect of no-flow boundary on normalized thickness

a positive permeability modulus and a large value of the sum modulus may give forewarning of future problems.

A reduced thickness may compromise well integrity. An altered permeability may impede or aid flow into the wellbore. There is no such thing as a comprehensive well test model for stress-sensitivity. Every reservoir is unique, and should ideally be treated as such. We believe our methodology will be useful since a field curve, obtained by a real test, may be matched against a model with both the modulus to thickness and permeability as unspecified variables. In case of a poor match, another method should be attempted. For example, this could be a numerical well test model. Then, any pressure function or even tabulated values may be used to describe the stress-sensitive variables.

In some cases, the duration of the test may be too short to detect neither an altered permeability nor a reduced thickness. There is still a chance that stress-sensitivity is an issue. Core analysis, laboratory experiments and well logging may add additional information. Then, the well test model may be used as an extrapolation tool with inferred values for the stress-sensitive variables.

The reliability of any model depends on how well the simplifying assumptions reflect the reality. The variation of permeability and porosity values with pressure has been investigated by laboratory experiments for decades. If we accept that these results provide insight at the length scale of well testing, then we have indirect information that can be used predict permeability and porosity. Some experiments for the permeability, show exponential like behavior, but the curves are shifted upwards compared to perfect exponential behavior, see figures based on core analysis in Kikani and Pedrosa (1991), Cao et al. (2004) and Jelmert et al. (2000). Since then, numerous authors have used the exponential model anyway. With inaccurate data, the computed response is valid for a limited pressure range only. For tight reservoirs, some cores show almost perfect exponential behavior for the permeability, Walls et al. (1982). In fact, this property was a key element to obtain unsteady state measurements of the permeability-pressure function. This method has the advantage of being much faster than the steady state method. The simplifying assumption that the bulk volume occupied by solid material is constant (Raghavan et al. 1972), leads to the conclusion that the variation of thickness depends on the change in porosity. Hence, the modulus to thickness may also be estimated from the porosity behavior by core analysis. Raghavan et al. (1972) presented core data to support their method. Due to the problem of upscaling, we believe data from core analysis is indicative only. Jelmert et al. (2000) suggested that an average value obtained from a set of cores, the geometric average, for example, might constitute a practical upscaling technique in lack of better alternatives.

Direct measurements of compaction in deep reservoirs are difficult and expensive. A method depends on the use of radioactive bullets. The method works best for vertical wells. For shallow reservoirs, it may be possible to observe compaction directly as wellheads protrude farther and farther from the surface, Doornhof et al. (2006).

The proposed technique is also available for partially penetrating wells and restricted entry problems Jelmert and Toverud (2017b).

Probably the best tool to analyze and predict compaction is numerical simulations, Doornhof et al. (2006). Such studies, however, are time consuming and expensive. A fluid extraction period may also be necessary for history matching. This period could be long for a tight reservoir. In addition, a numerical model cannot highlight the interaction between the variables by means of equations.

\section{Conclusions}

Reservoir compaction may have severe environmental ramifications, including subsidence, flooding and earthquakes. Pressure transient analysis has the potential to give the analyst an early warning against incipient problems. As such, the proposed method may be useful, at least for screening.

A well-known theory for pressure transient analysis in stress-sensitive formations has been generalized to account for simultaneous changes in thickness and permeability. Many scenarios give rise to the same predicted pressure signature, (non-uniqueness). Additional (independent) information is required to select the best reservoir model.

There is a possibility to estimate the modulus to thickness and thickness by type curve matching.

The reservoir permeability may increase because of new fractures generated. Then, the value of the permeability modulus could assume small and even negative values. This phenomenon masks real stress-sensitivity.

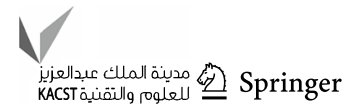


A positive permeability modulus highlights stress-sensitivity. A negative one has the opposite effect. The proposed model is unreliable in case of a negative sum modulus.

Acknowledgements The authors wish to express thanks to the Department of Geoscience and Petroleum for support permission to publish the present study.

Open Access This article is distributed under the terms of the Creative Commons Attribution 4.0 International License (http://creativeco mmons.org/licenses/by/4.0/), which permits unrestricted use, distribution, and reproduction in any medium, provided you give appropriate credit to the original author(s) and the source, provide a link to the Creative Commons license, and indicate if changes were made.

\section{Appendix A}

The following normalized variables are defined:

$$
\begin{aligned}
x_{n j}(p) & =e^{-\lambda_{j} \Delta p}, \quad x_{n j}=\frac{x_{j}}{x_{i}}, \quad j=1, \ldots, 6, \\
x_{j} & =k(p), \rho(p), \mu(p), \varphi(p), h(p), T(p), \\
\lambda_{j} & =\gamma, c, v, c_{\mathrm{ma}}, \xi, \tau .
\end{aligned}
$$

Index $n$ denotes normalized to initial condition. The latter is denoted by index $i$.

We assume a constant mass rate withdrawal.

$\dot{m}_{s f}=\rho_{s f} q_{s f}=\rho_{S c} q_{S c}=-\frac{2 \pi \rho_{i} k_{i} h_{i}}{\mu_{i}} \frac{\rho_{n}(\Delta p) k_{n}(\Delta p) h_{n}(\Delta p)}{\mu_{n}(\Delta p)} r \frac{\partial \Delta p}{\partial r}$.

Since the density, $\rho_{S c}$, is constant, then the rate of withdrawal, $q_{S c}$, is also constant.

Equation (20) will reduce to:

$\dot{m}_{s f}=\dot{m}_{s f i} \dot{m}_{s f n}$.

The flowrate is given by the non-linear Darcy's law. The assumption of exponential pressure functions leads to:

$q_{S c}=-\frac{2 \pi k_{i} h_{i} \rho_{i}}{\mu_{i} \rho_{S c}} e^{-\tau \Delta p} r \frac{\partial \Delta p\left(r_{w}\right)}{\partial r}=$ const.

Index $s f$ denotes sand face and $S c$ denotes standard conditions.

$\tau=\gamma+c+\xi-v$.

The density ratio is the inverse of the initial fluid formation factor, $B$.

We define a composite state variable, $T_{n}(\Delta p)$. Then:

$T_{n}(\Delta p)=\frac{k_{n}(\Delta p) h_{n}(\Delta p)}{\mu_{n}(\Delta p) B_{i}} \mathrm{e}^{-\tau \Delta p}$,

$q_{S c}=-2 \pi T_{i} T_{n}(\Delta p) r \frac{\partial \Delta p}{\partial r}$.
Solving Eq. (24) for the pressure change, $\Delta p=p_{i}-p$, we obtain

$\Delta p=-\frac{1}{\tau} \ln \left(T_{n}\right)=-\frac{1}{\tau} \ln \left(1-\Delta T_{n}\right)$

The spatial derivative of Eq. (26) becomes:

$\frac{\partial \Delta p}{\partial r}=-\frac{1}{\tau T_{n}} \frac{\partial T_{n}}{\partial r}$

Substitution Eq. (27) into Eq. (22) yields:

$q_{S c}=-\frac{2 \pi k_{i} h_{i}}{\mu_{i} B_{i} \tau} \cdot r \frac{\partial T_{n}\left(r_{w}\right)}{\partial r}=$ const.

The diffusivity equation for compacting media is, Raghavan et al. (1972):

$\frac{1}{r} \frac{\partial}{\partial r}\left(\dot{m}_{s f}\right)=\frac{\partial}{\partial \Delta p}(h(\Delta p) \rho(\Delta p) \varphi(\Delta p)) \frac{\partial \Delta p}{\partial t}$.

Substitution of Eq. (26) into Eq. (30) yields:

$\frac{1}{r} \frac{\partial}{\partial r}\left(\mathrm{e}^{-\tau \Delta p} r \frac{\partial \Delta p}{\partial r}\right)=\frac{\varphi_{i} \mu_{i}}{k_{i}} e^{-\left(\xi+c_{\ell}+c_{m a}\right) \Delta p}\left\{\xi+c_{\ell}+c_{m a}\right\} \frac{\partial \Delta p}{\partial t}$.

After some manipulations, we obtain:

$$
\begin{aligned}
& \frac{1}{r}\left\{\frac{\partial}{\partial r}\left(r \frac{\partial \Delta p}{\partial r}\right)-\tau r\left(\frac{\partial \Delta p}{\partial r}\right)^{2}\right\} \\
& \quad=\frac{\varphi_{i} \mu_{i}}{k_{i}} \mathrm{e}^{\left(\gamma+\xi-v+c_{\ell}-\left(\xi+c_{\ell}+c_{m a}\right)\right) \Delta p}\left\{\xi+c_{\ell}+c_{m a}\right\} \frac{\partial \Delta p}{\partial t} .
\end{aligned}
$$

At this point, we may proceed two ways; either evaluate exponential term at the initial condition, $\Delta p_{i}=0$, or use the Pedrosa method. By use of the first alternative, the exponential term becomes unity, which is the traditional pseudo pressure assumption. Then:

$\frac{1}{r}\left\{\frac{\partial}{\partial r}\left(r \frac{\partial \Delta p}{\partial r}\right)-\tau r\left(\frac{\partial \Delta p}{\partial r}\right)^{2}\right\}=\frac{\varphi_{i} \mu_{i}}{k_{i}}\left\{\xi+c_{\ell}+c_{m a}\right\} \frac{\partial \Delta p}{\partial t}$.

Pedrosa (1986) found that the perturbation scheme could be simplified whenever Eq. (31) can be approximated by Eq. (33),

$\frac{1}{r}\left\{\frac{\partial}{\partial r}\left(r \frac{\partial \Delta p}{\partial r}\right)-\tau r\left(\frac{\partial \Delta p}{\partial r}\right)^{2}\right\}=\frac{\varphi_{i} \mu_{i}}{k_{i}} e^{\tau \Delta p}\left\{\xi+c_{\ell}+c_{m a}\right\} \frac{\partial \Delta p}{\partial t}$.

The implied assumption is that:

$\gamma+\xi-v+c_{l}>>\xi+c_{\ell}+c_{m a}$ or $\quad \gamma>>v+c_{m a}$. 


\section{Appendix B}

We define dimensionless $\tau_{D}=\frac{q_{S c} \mu_{i} B_{i} \tau}{2 \pi k_{i} h_{i}}$ variables.

$r_{\mathrm{D}}=\frac{r}{r_{\mathrm{w}}}$

$t_{\mathrm{D}}=\frac{k_{i} t}{\varphi_{i} \mu_{i}\left(\xi+c_{\ell}+c_{m a}\right) r_{\mathrm{w}}^{2}}$,

$p_{\mathrm{D}}=\frac{2 \pi k_{i} h_{i}}{q_{S c} B_{i} \mu_{i}} \Delta p$

$\xi_{\mathrm{D}}=\frac{q_{S c} \mu_{i} B_{i} \xi}{2 \pi k_{i} h_{i}} \quad \gamma_{\mathrm{D}}=\frac{q_{S c} \mu_{i} B_{i} \gamma}{2 \pi k_{i} h_{i}} \quad c_{\mathrm{D}}=\frac{q_{S c} \mu_{i} B_{i} c}{2 \pi k_{i} h_{i}} \quad v_{\mathrm{D}}=\frac{q_{S c} \mu_{i} B_{i} v}{2 \pi k_{i} h_{i}}$,

$C_{\mathrm{D}}=\frac{C B_{i}}{2 \pi h_{i} \varphi_{i}\left(\xi+c_{\ell}+c_{m a}\right) r_{w}^{2}}$.

The variable, $C$, is the traditional wellbore storage constant.

The rate of withdrawal, $q_{S c}$, is an arbitrary reference rate at standard conditions..

Substitution of Eq. (35)-(38) into Eq. (33) yields:

$\frac{1}{r_{\mathrm{D}}}\left\{\frac{\partial}{\partial r_{\mathrm{D}}}\left(r_{\mathrm{D}} \frac{\partial p_{\mathrm{D}}}{\partial r_{\mathrm{D}}}\right)-\tau_{\mathrm{D}} r_{\mathrm{D}}\left(\frac{\partial p_{\mathrm{D}}}{\partial r_{\mathrm{D}}}\right)^{2}\right\}=e^{\tau_{\mathrm{D}} p_{\mathrm{D}}} \frac{\partial p_{\mathrm{D}}}{\partial t_{\mathrm{D}}}$

Substitution of Eq. (35)-(38) into Eq. (32) yields:

$\frac{1}{r_{\mathrm{D}}}\left\{\frac{\partial}{\partial r_{\mathrm{D}}}\left(r_{\mathrm{D}} \frac{\partial p_{\mathrm{D}}}{\partial r_{\mathrm{D}}}\right)-\tau_{\mathrm{D}} r_{\mathrm{D}}\left(\frac{\partial p_{\mathrm{D}}}{\partial r_{\mathrm{D}}}\right)^{2}\right\}=\frac{\partial p_{\mathrm{D}}}{\partial t_{\mathrm{D}}}$

Pedrosa (1986) proposed a logarithmic transformation:

$p_{\mathrm{D}}=-\frac{1}{\tau_{\mathrm{D}}} \ln \left(1-\tau_{\mathrm{D}} \eta\right)$.

This study is based on the above equation. This is because $\tau_{\mathrm{D}}$ may be used as a perturbation parameter. The generalized $T_{n}$ transformation (Eq. 26) becomes

$p_{\mathrm{D}}=-\frac{1}{\tau_{\mathrm{D}}} \ln \left(1-\Delta T_{n}\right)$.

Hence, all of Pedrosa's equations result from the following substitution:

$\eta=\frac{1}{\tau_{D}} \Delta T_{n}$

Substitution of Eq. (42) into Eq. (40) yields: $\frac{\partial^{2} \eta}{\partial r_{\mathrm{D}}^{2}}+\frac{1}{r_{\mathrm{D}}} \frac{\partial \eta}{\partial r_{\mathrm{D}}}=\frac{1}{1-\tau_{\mathrm{D}} \eta} \frac{\partial \eta}{\partial t_{\mathrm{D}}}$

Substitution of Eq. (42) into Eq. (41) yields Eq. (47).

We consider an infinite-acting reservoir for a fully penetrating line-source well. The boundary conditions become:

$\boldsymbol{\eta}\left(r_{\mathrm{D}}, 0\right)=0, \quad \lim _{r_{\mathrm{D}} \rightarrow \infty} \eta\left(r_{\mathrm{D}}, t_{\mathrm{D}}\right)=0, \quad \lim _{r_{\mathrm{D}} \rightarrow 0}\left(r_{\mathrm{D}} \frac{\partial \eta}{\partial r_{\mathrm{D}}}\right)=-1, \quad t_{\mathrm{D}} \geq 0$.

The perturbation scheme starts with the zero order solution, which has to satisfy Eq. (30), and Eq. (47).

$\frac{\partial^{2} \eta_{0}}{\partial r_{\mathrm{D}}^{2}}+\frac{1}{r_{\mathrm{D}}} \frac{\partial \eta_{0}}{\partial r_{\mathrm{D}}}=\frac{\partial \eta_{0}}{\partial t_{\mathrm{D}}}$

The zero order line-source solution at the sand-face is:

$\eta_{0}=\frac{1}{2} E_{1}\left(\frac{r_{\mathrm{D}}^{2}}{4 t_{\mathrm{D}}}\right)$

The first order solution is

$\eta_{1}=\frac{1}{2} E_{1}(2 z)-\frac{1}{4}\left(1+e^{-z}\right) E_{1}(z)$

$z=\frac{r_{\mathrm{D}}^{2}}{4 t_{\mathrm{D}}}$

The superposition function for build-up test becomes:

$\eta_{0 w s}(\Delta t)=\eta_{0 w}\left(t_{\mathrm{D}}+\Delta t_{\mathrm{D}}\right)-\eta_{0 w}\left(\Delta t_{\mathrm{D}}\right)$.

Which further becomes

$\eta_{0 w s}(\Delta t)=\frac{1}{2}\left(E_{1}\left(\frac{1}{4\left(t_{\mathrm{D}}+\Delta t_{\mathrm{D}}\right)}\right)-E_{1}\left(\frac{1}{4 \Delta t_{\mathrm{D}}}\right)\right)$,

and

$\eta_{0 w s}(\Delta t) \approx \frac{1}{2}\left(\ln \frac{t_{\mathrm{D}}+\Delta t_{\mathrm{D}}}{\Delta t_{\mathrm{D}}}\right)$

$p_{\mathrm{D} 0 w s}(\Delta t) \approx \frac{1}{\tau_{\mathrm{D}}}\left(1-\tau_{\mathrm{D}} \ln \frac{t_{\mathrm{D}}+\Delta t_{\mathrm{D}}}{\Delta t_{\mathrm{D}}}\right)$.

The traditional Horner equation is included in Eq. (54) as a limiting behavior.

$\lim \left(p_{\mathrm{D} w}\right)_{\tau_{\mathrm{D}} \rightarrow 0} \approx \frac{1}{2} \ln \frac{t_{\mathrm{D}}+\Delta t_{\mathrm{D}}}{\Delta t_{\mathrm{D}}}$. 


\section{References}

Al-Hussainy R, Ramey HJ, Crawford PB (1966) The flow of real gases through porous media. JPT. https://doi.org/10.2118/1243-A-PA

Cao W, Tong D, Wang R (2004) Exact solutions for nonlinear transient flow model including a quadratic gradient term. Appl Math Mech (Engl Ed) 25(1):102-109

Doornhof D, Kristiansen TG, Nagel NB, Patillo PD, Sayers C (2006) Compaction and subsidence. Oilfield Review, Autumn, pp 50-68

Friedel T, Voigt D (2009) Analytical solutions for the radial flow equation with constant-rate and constant-pressure boundary conditions in reservoirs with pressure sensitive permeability. In: SPE 122768, SPE rocky mountain petroleum technology conference, Denver, Colorado 14-16 April 1-19

The Guardian (2015) Shell and Exxon's $€ 5$ bn problem: gas drilling that sets off earthquakes and wreck homes

Jelmert TA, Selseng H (1997) Pressure transient behavior of stresssensitive reservoirs. In: SPE 38970, SPE Latin America/Caribbean conference, Rio de Janeiro, Brazil, 30 August-3 September

Jelmert TA, Toverud T (2016) Well test analysis by MDH type curves. IJETT 38(5):276-381

Jelmert TA, Toverud T (2017a) Compaction in a confined deforming reservoir. Energy Proc 128:165-171. http://www.sciencedirect. com

Jelmert TA, Toverud T (2017b) Approximate theory for well test interpretation of restricted entry wells in deforming reservoirs. In: 2nd international interdisciplinary conference on mineral waters, Vila De Luso, 26-31 March

Jelmert TA, Torsæter O, Selseng H (2000) Technique characterizes permeability of stress-sensitive reservoirs. Oil Gas J 52-61
Johnson AI ed (1991) Land subsidence, preface. IAHS Publication No. 200

Kikani J, Pedrosa OA (1991) Perturbation analysis in stress-sensitive formations. SPFE 6:379-386

Matthews CS, Brons F, Hazebrook P (1954) A method for determination of average pressure in bounded reservoirs. Trans AIME 201:182-191

Pedrosa OA (1986) Pressure transient in stress-sensitive formations. In: Proceedings of Latin American and Caribbean petroleum engineering conference, SPE 1515, 203-215

Prat WE, Johnson DW (1926) Local subsidence in the goose creek oil field. J Geol 34(7 Part 1):577-590

Raghavan R, Scoter JDT, Miller FG (1972) Investigation by numerical methods of the effect of pressure dependent rock and fluid properties on well flow tests. SPEJ 253:267-386

Samaniego F, Cinco-Ley H (1989) On the determination of the pressure-dependent characteristics of a reservoir trough transient pressure testing. In: SPE 19774, SPE annual technical conference and exhibition, San Antonio, 8-11 Oct, 9-20

The New York Times (1989) Jack-op project saves sinking North Sea rigs, Business

van Everdingen AF, Hurst W (1949) The application of the Laplace Transformation to flow problems in reservoirs. Petroleum Transactions, AIME, pp 305-324

Walls JD, Nur AM, Bourbie T (1982) Effects of pressure and partial water saturation on gas permeability in tight sands: experimental results. JPT 930-936

Publisher's Note Springer Nature remains neutral with regard to jurisdictional claims in published maps and institutional affiliations. 